
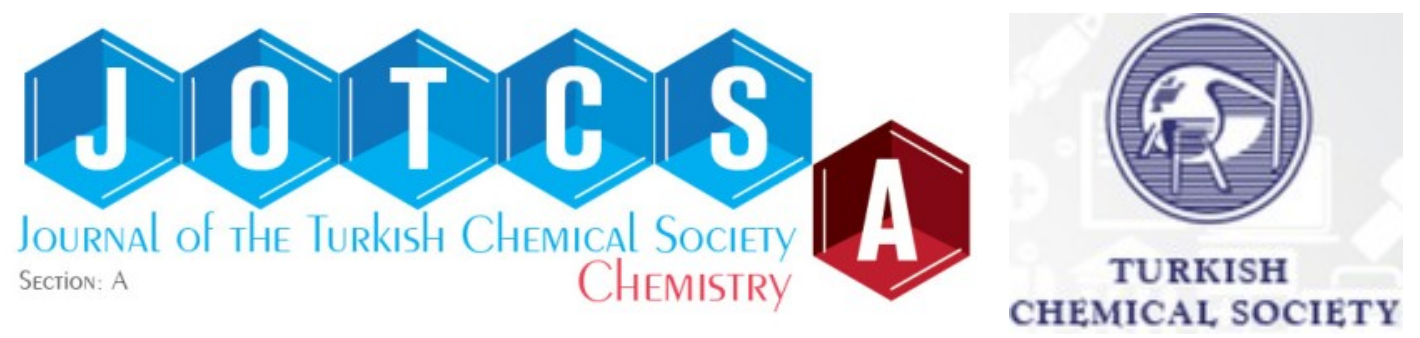

\title{
Kinetic Study of the Free Radical Copolymerization of Methyl Methacrylate with 2-Perfluorooctyl Ethyl Methacrylate by Quantum Computational Approach
}

\author{
Ramazan Katirci ${ }^{1}$ (D) and Salih Ozbay ${ }^{2}$ (D) \\ ${ }^{1}$ Department of Metallurgical and Materials Engineering, Sivas University of Science and Technology, 58000, \\ Sivas, Turkey \\ 2 Department of Chemical Engineering, Sivas University of Science and Technology, 58000, Sivas, Turkey
}

\begin{abstract}
Fluorinated copolymers with perfluoroalkyl side chains have widespread use in applications requiring superior technology due to their unique surfacial properties. Kinetic analysis of copolymerization of fluorinated acrylates with conventional acrylates is necessary to synthesize such copolymers efficiently. However, kinetic investigation of such reactions are limited in the literature due to the experimental difficulties. In this study, the kinetics of copolymerization of methyl methacrylate with 2-perfluorooctyl ethyl methacrylate in toluene medium using AIBN initiator was investigated using quantum chemistry postulates as an alternative to experimental methods. Reaction rate constants $\left(k_{\mathrm{p}}\right)$ for propagation were determined using transition state theory. A terminal effect models were used to examine four different addition reactions involving monomeric and dimeric radicals and monomers for both self- and cross-propagation. Reactant and product conformations were optimized with a DFT method using PBEO function. The Evans-Polanyi relationship was used to calculate the rate of self- and cross-propagation of monomers. The results showed that the reactivity ratio of 2-perfluorooctyl ethyl methacrylate was found to be higher than that of methyl methacrylate. In addition, it was observed that the reaction conditions caused the random polymer structure due to the different rate constants in self and cross propagation.
\end{abstract}

Keywords: 2-Perfluorooctyl ethyl methacrylate, methyl methacrylate, free radical polymerization, kinetics, DFT

Submitted: May 30, 2021. Accepted: November 09, 2021.

Cite this: Katirci R, Ozbay S. Kinetic Study of the Free Radical Copolymerization of Methyl Methacrylate with 2Perfluorooctyl Ethyl Methacrylate by Quantum Computational Approach. JOTCSA. 2021;8(4):1263-74.

DOI: https://doi.org/10.18596/jotcsa.945056.

*Corresponding author. E-mail: salihozbay@sivas.edu.tr. Phone: +90 (346) 2191398.

\section{INTRODUCTION}

Fluorinated polymers have widespread applications in both scientific studies and industry due to their unique surface properties such as oil/water repellency originated from low polarizability and high electronegativity of the fluorine atoms (1-6). Design of superhydrophobic $(7,8)$ superoleophobic $(9,10)$ or superamphiphobic (11-13) surfaces using fluoropolymers is a common and practical way. Perfluorinated (meth)acrylates (FMA) are an important member of fluoropolymers because of their extremely low surface free energy properties (14-17).
However, adaptation of homopolymers of FMA to many systems is limited due to the many drawbacks such as being expensive, having poor mechanical properties and solubility difficulties in organic solvents $(3,13,18)$. Copolymerization of FMA with methyl methacrylate (MMA) is an efficient solution to overcome these problems, and FMA-MMA copolymer couples have been experimentally synthesized by free radical polymerization using thermal initiators many times in the literature under different reaction conditions $(15,18-28)$. Organic solvents such as butyl acetate $(19,20,23)$, methyl ethyl ketone (MEK) (7, 21), cyclohexanone (24), toluene $(29,30)$ and solvent mixtures such as 1,1,2- 
trichlorotrifluoroethane (R-113)/ $\alpha, \alpha, \alpha$-trifluorotoluene (TFT) $(15,18)$, toluene/MEK (25) were previously used in solution copolymerizations to synthesize fluorinated acrylate copolymers. Apart from conventional organic solvents, supercritical $\mathrm{CO}_{2}$ has been used in many studies as an alternative medium for the free radical polymerization of fluoro monomers (26-28). Although these studies were successful in understanding the reaction conditions to obtain such polymers, and to find out the bulk and surface properties of the synthesized polymer, additional studies are needed for a detailed kinetic examination.

The kinetics of free radical copolymerization are critical for controlling the polymerization process and the last product (31). In a copolymerization where two different monomers are bonded to each other covalently, there are four divergent possible combinations of monomers and radical ends, and there is no experimental method that can directly measure the individual rate constant $\left(k_{\mathrm{p}}\right)$ values for different combinations of monomers and radicals (32). In addition, the determination of the overall $k_{\mathrm{p}}$ is also difficult by experimental methods due to the reactivity differences between secondary and tertiary carbon radicals $(33,34)$. Whereas, quantum computational approaches which does not require experimental investigations provides convenience to determine many reaction parameters and molecular structure properties such as the rate constant $\left(k_{\mathrm{p}}\right)$, activation energy $\left(E_{a}\right)$, Gibbs free energy difference, $\Delta G$, transition state geometries, and molecular architecture.

In this study, we investigated the kinetic of copolymerization of 2-perfluorooctyl ethyl methacrylate (FOEMA) with MMA by quantum computational approaches. Copolymers of FOEMA with MMA were synthesized computationally using different monomer feed compositions by a free radical process in toluene medium. AIBN was used as a thermal initiator. The main aim of this study is to find out the propagation rate in the intermediate steps and find the step determining the reaction rate, which provide us to estimate the sequence of the polymerization. In addition, the reactivity ratios were determined for the copolymerization of MMA with FOEMA to evaluate feed/bulk composition balance. A computational methodology was used to study such acrylate polymerization reactions, which is adaptable to other fluorinated or non-fluorinated acrylate polymerization systems.

\section{COMPUTATIONAL METHODOLOGY}

Four addition reactions of monomeric and dimeric radicals to monomers in all possible different combinations were studied for MMA and FOEMA as shown in Tables 1 and 2 . For all reactions, $k_{\mathrm{p}}$ were calculated individually. The $\Delta G$ for these reactions was calculated at $350 \mathrm{~K}$ and 1 atm pressure to acquire the reaction rates of free radical polymerizations using the Evans-Polanyi equation (32) as shown in Equation (1),

$$
k_{p}=\frac{k_{B} T}{h c^{\circ}} e^{-\Delta \ddagger G^{\circ} / R T}
$$

where $k_{\mathrm{B}}$ denotes Boltzmann's constant $\left(1.3806 \times 10^{-}\right.$ $23 \mathrm{~J} / \mathrm{K}) ; h$ is Planck's constant $\left(6.6261 \times 10^{-34} \mathrm{~J} /\right.$ mol.K); $c^{\circ}$ standard state concentration (mol/L), which can be taken as 1 , and $\Delta G^{\circ}$ is the free energy difference between the activated complex and the reactants (with inclusion of zero point vibration energies). G energy is associated with a chemical reaction which can be used to do a work.

The preliminary study was performed to identify the best functional and basis set compatible with the experimental study. In the preliminary study, the optimization and frequency calculations were carried out with M062X/6-31G(d,p), B3LYP/6-31G(d,p), PBE/6-31G(d,p) and PBE0/def2-TZVP functional and basis sets. The best results were acquired with PBE0/def2-TZVP method and we continued our computations with this method. The results of other method were presented in the supporting information file (Table S3). All electronic energy calculations and vibrational frequencies were calculated using Orca 4.2 $(35,36)$. All the reactants and product conformations were optimized using DFT method and PBEO function with def2-TZVP basis set (37) at $350 \mathrm{~K}$ and 1 atm pressure. However, the conventional optimization method employed is based on the gradient in energy and can only locate local minima, and thus the optimized geometry is sensitive to the input structure. Therefore, different conformations were explored using a systematic rotor search method which is present in Avogadro software. The conformations having the lowest energy were used as the input.

Transition state (TS) structures were screened using the relaxed scan method in Orca 4.2 software. The geometry owning the lowest energy in TS mode was used as the optimized TS geometry. Transition states were confirmed to have one imaginary frequency, which corresponded to the motion along the reaction coordinate, and an intrinsic reaction coordinate (IRC) (32) was performed to verify that the correct reactants and product were obtained. The conductorlike polarizable continuum model (CPCM) was used to calculate the solution effect (36).

It is critical to have a quantum chemical calculation method/basis set which is accurate yet computationally affordable due to the size of the polymer structures which were studied in this work. In many studies, it was reported that the activation energies and $k_{\mathrm{p}}$ values obtained using the PBEO function with the basis set of def2-TZVP was a good enough agreement with experimental data (38). 


\section{RESULTS AND DISCUSSION}
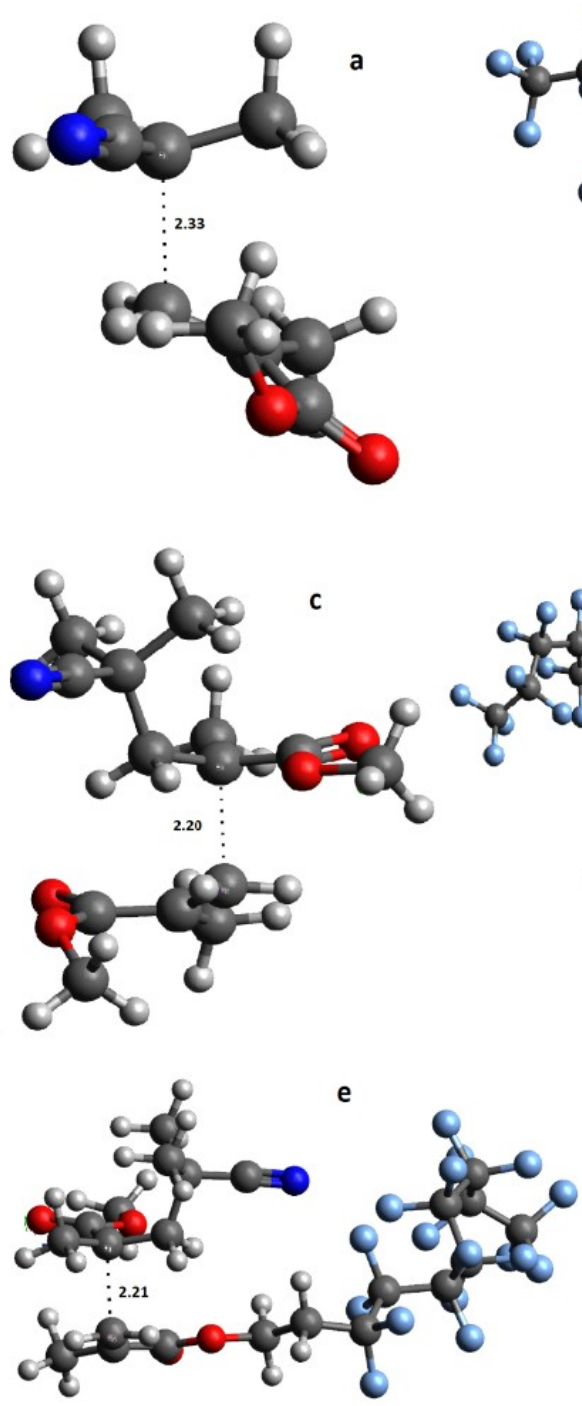

The TS geometries of the fluorinated acrylate molecules are presented in Figure 1.
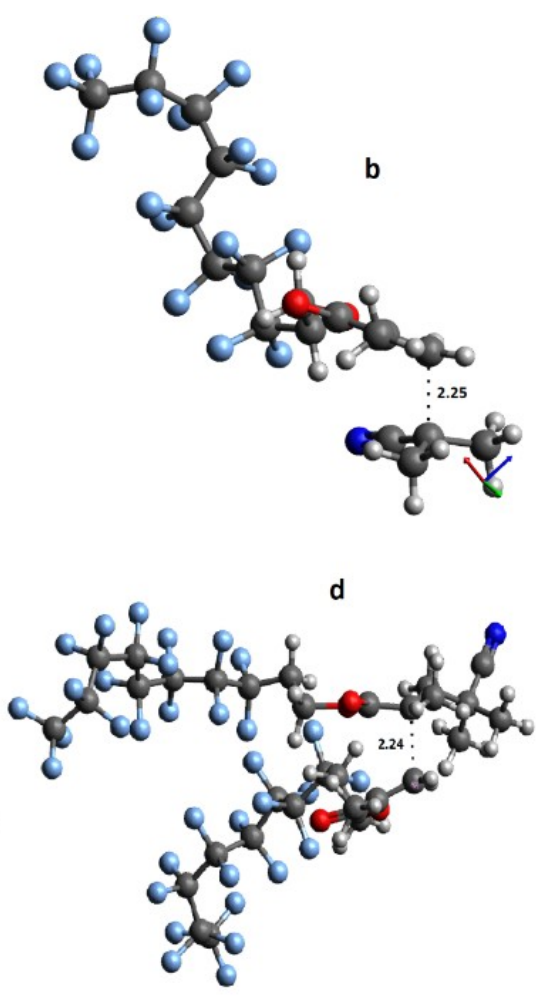

Figure 1: Transition state geometries of a) R-MMA b) R-FOEMA c) R-MMA-MMA d) R-FOEMA-FOEMA e) RMMA-FOEMA f) R-FOEMA-MMA (PBE0/ def2-TZVP, $1 \mathrm{~atm}, 350 \mathrm{~K}$, toluene medium (CPCM)).

The geometry of the transition states, which shows the minimum energy for the reaction formation, gives the significant information about combinations of fluorine atoms. The difference between the energies of the transition and the initial states determine the experimental activation energy for the reaction (39). The geometry of TS has the highest energy along the reaction coordinate and more free energy in comparison to the substrate or product; thus, it is the least stable state. Reactants, products and an estimation of the transition states are required to locate the transition state structures. Chemical structures of reactants and representative reaction of MMA, FOEMA and AIBN are given in Figure 2. The addition of the radical center to the unsaturated $\mathrm{C}=\mathrm{C}$ bond of the monomer is shown in Figure 3. The distance between the addition monomer and radical in the transition state was varied in the range of 2.202.33. These distances indicate that they have the partial bond at their maximum length. Because the lifetime of the transition state is too short and rapidly relaxes to the product, it is too difficult to determine the $k_{\mathrm{p}}$ values experimentally. Transition states are specified as saddle points on the potential energy surface, possessing one imaginary frequency. Once possible transition state locations were acquired, they were confirmed using intrinsic reaction coordinate (IRC) (40). 
<smiles>CC(C)(C)N=NC(C)(C)C#N</smiles>

AIBN

R

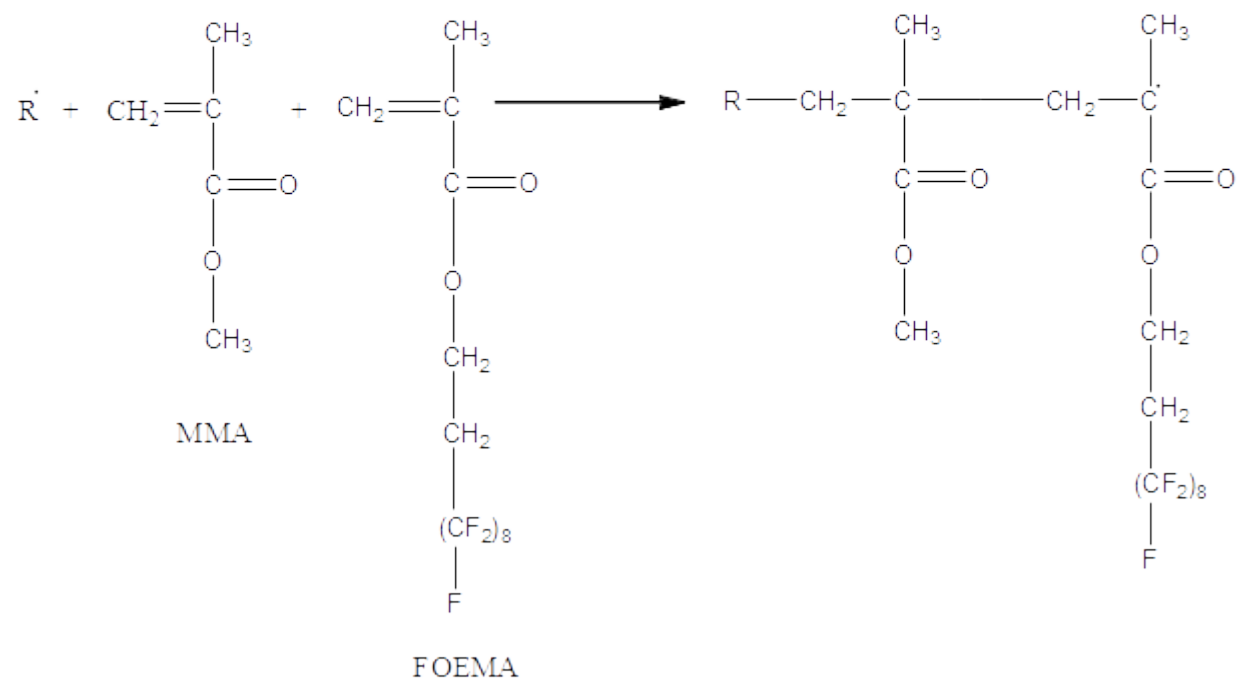

Figure 2. Representative reaction of methyl methacrylate (MMA), 2-perfluorooctyl ethyl methacrylate (FOEMA) and 2,2'-azobisisobutyronitrile (AIBN).

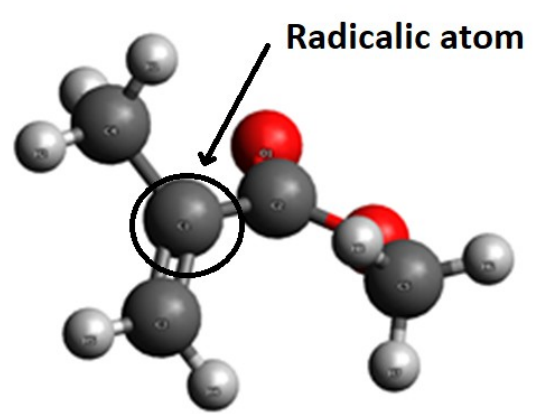

Figure 3. The geometric structure of MMA monomer (PBE0/ def2-TZVP, $1 \mathrm{~atm}, 350 \mathrm{~K}$, toluene medium $(\mathrm{CPCM}))$.

Table 1. Relative Gibbs free energy of monomers, radicals, and transition states (TS) of molecules (PBEO/ def2-TZVP, $1 \mathrm{~atm}, 350 \mathrm{~K}$, toluene medium (CPCM)).

\begin{tabular}{cc}
\hline & Gibbs Free Energy $(\mathrm{kcal} / \mathrm{mol})$ \\
\hline $\mathrm{RA}^{\bullet}$ (Radical) + A (Monomer) & 0 \\
$\mathrm{RB}^{\bullet}$ (Radical) + B (Monomer) & 0 \\
$\mathrm{RA}^{\bullet}$ (Radical) + B (Monomer) & 0 \\
$\mathrm{RB}^{\bullet}$ (Radical) + A (Monomer) & 0 \\
RA -- A (TS) & 16.5 \\
RB -- B (TS) & 16.5 \\
RA -- B (TS) & 16.3 \\
RB -- A (TS) & 16.8 \\
\hline
\end{tabular}

Table 2. Frequency factors $(A)$, Activation energies $\left(E_{\mathrm{a}}\right)$ and rate constants for the MMA-FOEMA polymerizations (PBE0/ def2-TZVP, $1 \mathrm{~atm}, 350 \mathrm{~K}$, toluene medium (CPCM)). 


\begin{tabular}{cccc}
\hline Polymerizations & $\log _{10}(A)$ & $E_{\mathrm{a}}(\mathrm{kcal} / \mathrm{mol})$ & $k_{\mathrm{p}}\left(\mathrm{s}^{-1}\right)$ \\
\hline $\mathrm{RA}^{\bullet}+\mathrm{A} \rightarrow \mathrm{RAA}^{\bullet}$ & 3.32 & 1.20 & $3.688 \times 10^{2}$ \\
$\mathrm{RA}^{\bullet}+\mathrm{B} \rightarrow \mathrm{RAB}^{\bullet}$ & 3.23 & 0.90 & $4.681 \times 10^{2}$ \\
$\mathrm{RB}^{\bullet}+\mathrm{B} \rightarrow \mathrm{RBB}^{\bullet}$ & $*$ & $*$ & $3.877 \times 10^{2 *}$ \\
$\mathrm{RB}^{\bullet}+\mathrm{A} \rightarrow \mathrm{RBA}^{\bullet}$ & 2.57 & 0.28 & $2.497 \times 10^{2}$ \\
\hline
\end{tabular}

$E_{a}$ : Activation Energy. A: The frequency or pre-exponential factor. $k_{p}$ : Rate constant. " $R$ " stands for 2,2 'azobisisobutyronitrile, "A" denotes methyl methacrylate (MMA), and " $B$ " denotes perfluorooctyl ethyl methacrylate (FOEMA). *The Gibbs free energy of TS in this reaction was computed as -4980.71 hartree. But the $k_{p}$ value in Table 2 was calculated according to the number of 4980.73 (Table S4). We searched many conformations to find the global minima, but we could not reach the global minimum conformation. The computations took too much cpu time and large memory because the structure of the molecule was too large. Because the other $k_{p}$ values were compatible with the experimental studies, we decided to report by estimating the Gibbs free energy of TS of RB- - $\mathrm{B}^{*}$.

Table 1 shows the Relative Gibbs free energy (G) of the reactants and TS molecules. The $k_{\mathrm{p}}$ constants and kinetic parameters ( $A, E a)$ of the polymerizations in Table 2 were computed using the data in supporting information Table S4. Reaction rate constants $\left(k_{\mathrm{p}}\right)$ for propagation were computed using equation (1) (41). The calculations were carried out in toluene and gas phase. In the gas phase, the $k_{\mathrm{p}}$ constants were too low $\left(k_{\mathrm{p}}=\sim 10^{-30}-10^{-50}\right)$, so it can be said that the polymerization reaction was not possible in the gas phase. The results in the gas phase were presented in supporting information Table S1 and Table S2. When the toluene as the solution was included in the calculations, the $k_{\mathrm{p}}$ constants raised abruptly, which means the solution effect is very high. The CPCM model was implemented to compute the solution effect. In this model, the solvent is represented as a dielectric polarizable continuum and the solute is located in a cavity of approximately the molecular shape. The solvent reaction area is described by polarization charges on the surface of the cavity. The cavity is generated by the GEPOL algorithm using a solvent-excluding or solvent-accessible surface.

The monomer reactivity ratios of $r_{\mathrm{A}}$ and $r_{\mathrm{B}}$ were computed using the Equations (2) and (3).

$$
\begin{aligned}
& r_{A}=\frac{k_{A-A}}{k_{A-B}} \\
& r_{B}=\frac{k_{B-B}}{k_{B-A}}
\end{aligned}
$$

According to the calculations, $r_{\mathrm{A}}$ and $r_{\mathrm{B}}$ were estimated as 0.79 and 1.55 , respectively indicating that the fluorinated acrylate monomer is much more reactive than MMA monomer. Reactivity ratio differences between fluorinated acrylate and MMA can be seen in the previous experimental reports considering reactivity ratio values or feed/bulk composition balance (19, 20, 24-28). For example, van de Grampel et al. reported $r_{\mathrm{MMA}}=0.76$ and $r_{\text {fluoro }}$ methacrylate $=1.31$ for poly(MMA-co-1,1dihydroperfluoroheptyl methacrylate) copolymer system using ${ }^{1} \mathrm{H}-\mathrm{NMR}$ data and nonlinear leastsquares data fitting (19). The reactivity ratios can also be used to evaluate the composition in the copolymer product as a function of monomer feed fractions based on Mayo-Lewis equations (42) as shown in Equation (4) (43),

$$
F_{1}=\frac{r_{1} f_{1}^{2}+f_{1} f_{2}}{r_{1} f_{1}^{2}+2 f_{1} f_{2}+r_{2} f_{2}^{2}}
$$

where $F$ denotes a molar fraction of monomer in the copolymer, $f$ denotes molar fraction of monomers in the feed and $r$ denotes reactivity ratios. Using the reactivity ratio values and equation (4) simultaneously, Mayo-Lewis plot of FFOEMA vs $f_{\text {FOEMA }}$ was constructed and is shown in Figure 4. The predicted composition curve is in good accordance with the previous experimental reports associated with the perfluoro acrylate - MMA copolymerization systems, indicating that our approach is easily able to adaptable to the real copolymerization systems. 


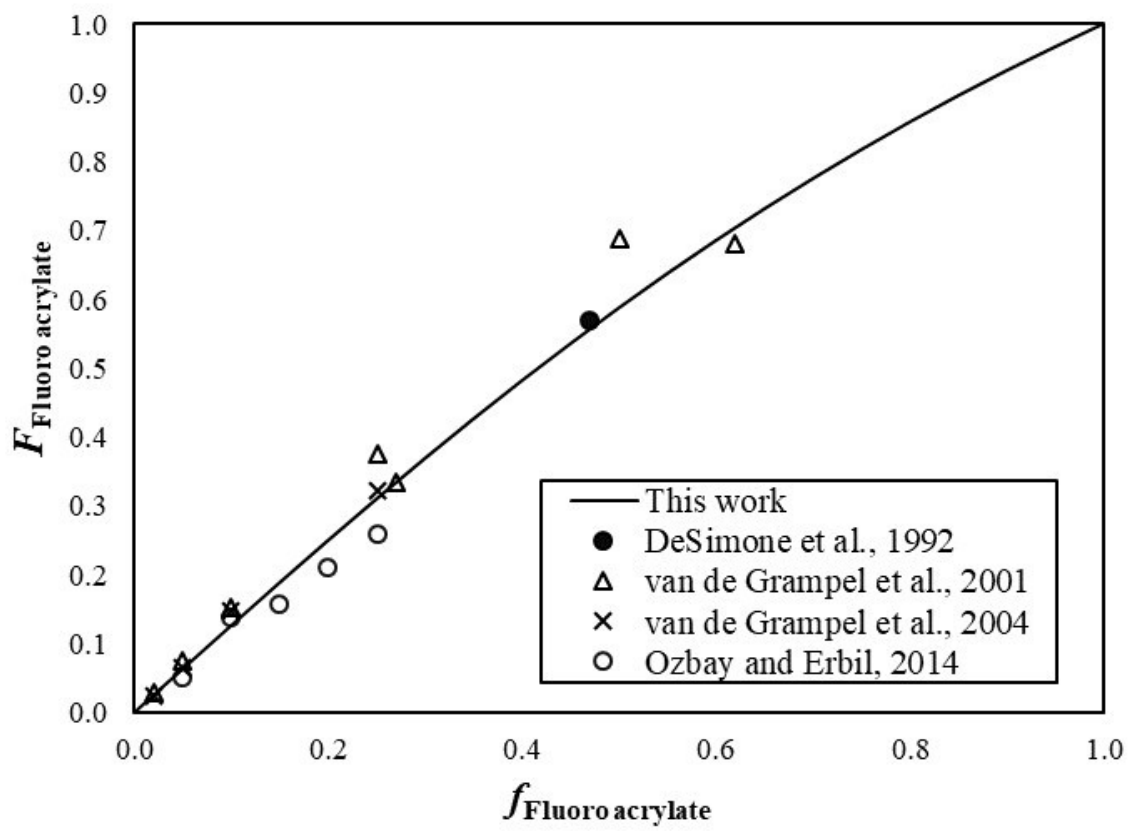

Figure 4. Mayo-Lewis plot for MMA-FOEMA copolymerization at $350 \mathrm{~K}$ and $1 \mathrm{~atm}$. Copolymer composition data for poly(1,1-dihydroperfluorooctyl acrylate-co-MMA) copolymerization synthesized in supercritical $\mathrm{CO}_{2}$ medium at $332.55 \mathrm{~K}(\bullet)(26)$; for poly(1,1-dihydroperfluoroheptyl methacrylate-co-MMA) copolymerization synthesized in butyl acetate at $353.15 \mathrm{~K}(\Delta)$ (19); for poly(1,1-dihydroperfluoroheptyl methacrylate-co-MMA) copolymerization synthesized in butyl acetate at $353.15 \mathrm{~K}(\times)(20)$; poly(perfluoroalkyl ethyl methacrylate-coMMA) synthesized in toluene/MEK solvent mixture at $348.15 \mathrm{~K}$ (0) (25).

On the other hand, linear copolymers can be in random, block, or alternative copolymer structure depending on the arrangement of their monomers. DSC analysis is the most practical experimental way to say that a copolymer is random. If a copolymer shows one glass transition peak, this polymer can be considered as random copolymer. In general, copolymerization of acrylates with fluorinated acrylates by using thermal initiators gives random copolymer structure. For example, Chang et al. synthesized fluoroacrylate-MMA random copolymer in MEK solvent using AIBN initiator at $70{ }^{\circ} \mathrm{C}$ (7). Similarly, Nishino et al. synthesized 2perfluorooctylethyl methacrylate-MMA random copolymer in MEK solvent using AIBN initiator at 70 ${ }^{\circ} \mathrm{C}$ (21). Park et al. synthesized perfluoroalkylethyl methacrylate-MMA random copolymer in R-113/TFT solvent mixture using AIBN initiator at $70^{\circ} \mathrm{C}(15,18)$. $\mathrm{Ye}$ et al. synthesized 2-perfluorooctylethyl methacrylate-MMA random copolymer in cyclohexanone solvent using benzoyl peroxide (BPO) initiator at $70{ }^{\circ} \mathrm{C}(24)$. Ozbay and Erbil synthesized 2perfluoroalkylethyl methacrylate-MMA random copolymers in toluene/MEK solvent mixture using AIBN initiator at $75^{\circ} \mathrm{C}$ (25). All of these results experimentally indicate that free radical copolymerization of fluorinated acrylates with conventional acrylates using thermal initiators caused random copolymer structure. However, the computational proof of this experimental result is another case. The rate constant of a reaction can be used to estimate the molecular architecture of copolymer. For example, it is expected that the reactivity ratios $\left(r_{\mathrm{A}}\right.$ and $r_{\mathrm{B}}$ ) of all monomers should be equal to or close to zero for an alternating copolymer. When the $k_{\mathrm{p}}$ values are examined in Table 2, it is seen that they are quite different from each other. Therefore, it can be said that the alternating copolymer is not possible in these reactions. If we make this evaluation for a block copolymer, the reactivity ratios of both monomers should be higher than 1 . In our study, the $r_{\mathrm{A}}$ value is lower than 1 . This indicates that the formation of block copolymer is not possible in these reactions. However, in a random copolymer, the two monomers may react in any order. The proportion of the monomers added into the copolymer is a result of a combination of the properties of the monomers, the polymerization conditions and the conversion of the polymerization. For example, if the two monomers do not have the same reactivity exactly, the ratio in the product will not occur exactly 1-to-1. It results in a change in the copolymer composition while the reaction proceeds. At the beginning, the more reactive monomer is added more than the less reactive one. When the $k_{\mathrm{p}}$ values are examined in Table 2, the reactivity of the polymer chain is different. Thus, it can be deduced that the polymer reaction proceeds randomly. This result is compatible with the experimental studies carried out by various researchers $(20,21-28)$. 


\section{CONCLUSIONS}

A computational methodology based on quantum postulates and transition state theory has been used to estimate the kinetic parameters of MMA and FOEMA propagation reactions. A conventional geometric optimization method was used to locate the global minimum geometry of the monomers. These monomers were added in a different order. All reactants and products were optimized. To determine the transition state, relaxed potential energy scans were carried out. The bond defining the transition state was used to estimate the Gibbs free energy in the highest energy along the reaction coordinate. IRC calculations were performed to verify the location of the transition state. The $k_{\mathrm{p}}$ constants were determined at $350 \mathrm{~K}$ for free radical copolymerization in toluene medium. The reactivity ratios for the free radical copolymerization of MMA and FOEMA in toluene medium were determined to be $r_{\mathrm{MMA}}=0.79$ and $r_{\text {FOEMA }}=1.55$. It was observed that predicted reactivity ratios and feed/bulk composition balance are in good accordance with the previous experimental reports, indicating that our approach is easily able to adaptable to the various copolymerization systems.

\section{REFERENCES}

1. Ciardelli F, Aglietto M, Montagnini di Mirabello L, Passaglia E, Giancristoforo S, Castelvetro V, et al. New fluorinated acrylic polymers for improving weatherability of building stone materials. Progress in Organic Coatings. 1997;32(1-4):43-50. <DOI .

2. Anton D. Surface-fluorinated coatings.". Advanced Materials. 1998;10:1197-205.

3. Imae T. Fluorinated polymers. Current Opinion in Colloid \& Interface Science. 2003;8(3):307-14. <DOI>.

4. Huang $\mathrm{P}-\mathrm{Y}$, Chao $\mathrm{Y}-\mathrm{C}$, Liao $\mathrm{Y}-\mathrm{T}$. Preparation of fluoroacrylate nanocopolymer by miniemulsion polymerization used in textile finishing. Journal of Applied Polymer Science. 2004;94(4):1466-72. $<$ DOI $>$.

5. Bruno A. Controlled Radical (Co)polymerization of Fluoromonomers. Macromolecules. 2010;43(24):10163-84. <DOI>.

6. Lee S, Park J-S, Lee TR. The Wettability of Fluoropolymer Surfaces: Influence of Surface Dipoles. Langmuir. 2008;24(9):4817-26. <DOI>.

7. Chang $\mathrm{K}-\mathrm{C}$, Chen $\mathrm{H}$, Huang C-K, Huang S-I. Preparation of super-hydrophobic film with fluorinated-copolymer. Journal of Applied Polymer Science. 2007;104(3):1646-53. <DOI>.

8. Ozbay S, Erbil HY. Superhydrophobic and oleophobic surfaces obtained by graft copolymerization of perfluoroalkyl ethyl acrylate onto SBR rubber. Colloids and Surfaces A: Physicochemical and Engineering Aspects. 2015;481:537-46. <DOI>.

9. Tuteja A, Choi W, Ma M, Mabry JM, Mazzella SA, Rutledge GC, et al. Designing superoleophobic surfaces. Science. 2007;318(5856):1618-22. <DOI>.

10. Steele A, Bayer I, Loth E. Inherently Superoleophobic Nanocomposite Coatings by Spray Atomization. Nano Letters. 2009;9(1):501-5. <DOI>.

11. Xiong D, Liu G, Hong L, Duncan EJS. Superamphiphobic Diblock Copolymer Coatings. Chemistry of Materials. 2011;23(19):4357-66. $\leq$ DOI $>$.

12. Lee SG, Ham DS, Lee DY, Bong $H$, Cho $\mathrm{K}$. Transparent Superhydrophobic/Translucent Superamphiphobic Coatings Based on SilicaFluoropolymer Hybrid Nanoparticles. Langmuir. 2013;29(48):15051-7. <DOI>.

13. Ozbay S, Cengiz U, Erbil HY. Solvent-Free Synthesis of a Superamphiphobic Surface by Green Chemistry. ACS Applied Polymer Materials. 2019;1(8):2033-43. <DOI>.

14. Katano $\mathrm{Y}$, Tomono $\mathrm{H}$, Nakajima $\mathrm{T}$. Surface Property of Polymer Films with Fluoroalkyl Side Chains. Macromolecules. 1994;27(8):2342-4. <DOI>.

15. Park IJ, Lee S-B, Choi CK. Surface Properties of the Fluorine-Containing Graft Copolymer of Poly((perfluoroalkyl)ethyl methacrylate)- g poly(methyl methacrylate). Macromolecules. 1998;31(21):7555-8. <DOI>.

16. Stone M, Nevell TG, Tsibouklis J. Surface energy characteristics of poly(perfluoroacrylate) film structures. Materials Letters. 1998;37(1-2):102-5. $<$ DOI $>$.

17. Tsibouklis J, Graham P, Eaton PJ, Smith JR, Nevell TG, Smart JD, et al. Poly(perfluoroalkyl methacrylate) Film Structures: Surface Organization Phenomena, Surface Energy Determinations, and Force of Adhesion Measurements. Macromolecules. 2000;33(22):8460-5. <DOI>

18. Park IJ, Lee S-B, Choi CK. Synthesis of fluorine-containing graft copolymers of poly(perfluoroalkylethyl methacrylate)-g-poly(methyl 
methacrylate) by the macromonomer technique and emulsion copolymerization method. Polymer. 1997;38(10):2523-7. <DOI>.

19. Van De Grampel RD, Van Geldrop J, Laven J, Van Der Linde R. P[CF3(CF2)5CH2MA-Co-MMA] and P[CF3(CF2)5CH2MA-Co-BA] copolymers: Reactivity ratios and surface properties. Journal of Applied Polymer Science. 2001;79(1):159-65. <DOI>.

20. Van de Grampel RD, Ming W, Gildenpfennig A, Van Gennip WJH, Laven J, Niemantsverdriet JW, et al. The Outermost Atomic Layer of Thin Films of Fluorinated Polymethacrylates. Langmuir. 2004;20(15):6344-51. <DOI>.

21. Nishino $T$, Urushihara $Y$, Meguro $M$, Nakamae $\mathrm{K}$. Surface properties and structures of diblock and random copolymers with perfluoroalkyl side chains. Journal of Colloid and Interface Science. 2004;279(2):364-9. <DOI>.

22. Choi D, Yeom EH, Park M, Kim JK, Kim BC. Preparation and properties of methyl methacrylate and fluoroacrylate copolymers for plastic optical fiber cladding. Journal of Applied Polymer Science. 2004;93(5):2082-9. <DOI>.

23. Hartmann $P$, Collet $A$, Viguier $M$. Acrylic Copolymers with Perfluoroalkylated Biphenyl Side Groups: Correlation Structure-Surface Properties. Macromolecules. 2006;39(20):6975-82. <DOI>.

24. Ye X, Zuo B, Deng M, Hei Y, Ni H, Lu X, et al. Surface segregation of fluorinated moieties on poly(methyl methacrylate-ran-2-perfluorooctylethyl methacrylate) films during film formation: Entropic or enthalpic influences. Journal of Colloid and Interface Science. 2010;349(1):205-14. <DOI>.

25. Ozbay S, Erbil HY. Solution copolymerization of perfluoroalkyl ethyl methacrylate with methyl methacrylate and butyl acrylate: Synthesis and surface properties. Colloids and Surfaces A: Physicochemical and Engineering Aspects. 2014;452:9-17. <DOI>.

26. DeSimone AJM, Guan Z, Elsbernd CS. Synthesis of Fluoropolymers in Supercritical Carbon Dioxide. Science. 1992;257(5072):945-7.

27. Cengiz U, Gengec NA, Ugur Kaya N, Yildirim Erbil H, Sezai Sarac A. Mechanical and thermal properties of perfluoroalkyl ethyl methacrylatemethyl methacrylate statistical copolymers synthesized in supercritical carbon dioxide. Journal of Fluorine Chemistry. 2011;132(5):348-55. <DOI>.
28. Cengiz U, Gengec NA, Erbil HY. Surface characterization of flat and rough films of perfluoromethacrylate-methylmethacrylate statistical copolymers synthesized in CO2-expanded monomers. Colloid and Polymer Science. 2013;291(3):641-52. $\leq$ DOI $>$.

29. Ding L, Olesik S V. Dispersion Polymerization of MMA in Supercritical CO 2 in the Presence of Copolymers of Perfluorooctylethylene Methacrylate and Poly(propylene glycol) Methacrylate. Macromolecules. 2003;36(13):4779-85. <DOI>.

30. Valtola L, Hietala S, Tenhu H, Denifl P, Wilen C-E. Association behavior and properties of copolymers of perfluorooctyl ethyl methacrylate and eicosanyl methacrylate. Polymers for Advanced Technologies. 2009;20(3):225-34. <DOI $>$.

31. Fukuda T, Kubo K, Ma Y-D. Kinetics of free radical copolymerization. Progress in Polymer Science. 1992;17(5):875-916. <DOI>.

32. Yu X, Levine SE, Broadbelt L. Kinetic Study of the Copolymerization of Methyl Methacrylate and Methyl Acrylate Using Quantum Chemistry. Macromolecules. 2008;41(21):8242-51. 〈DOI>.

33. Asua JM, Beuermann $S$, Buback $M$, Castignolles P, Charleux B, Gilbert RG, et al. Critically Evaluated Rate Coefficients for Free-Radical Polymerization, 5,. Macromolecular Chemistry and Physics. 2004;205(16):2151-60. <DOI>.

34. Dossi M, Storti G, Moscatelli D. A quantum chemistry study of the free-radical copolymerization propagation kinetics of styrene and 2-hydroxyethyl acrylate. Polymer Engineering \& Science. 2011;51(10):2109-14. <DOI>.

35. Neese F. The ORCA program system. WIREs Computational Molecular Science. 2012;2(1):73-8. $\leq \mathrm{DOI}>$.

36. Neese F. Software update: the ORCA program system, version 4.0. WIREs Computational Molecular Science. 2018;8:e1327. <DOI>.

37. Treutler O, Ahlrichs R. Efficient molecular numerical integration schemes. The Journal of Chemical Physics. 1995;102(1):346-54. <DOI>.

38. Liu S, Srinivasan S, Tao J, Grady MC, Soroush M, Rappe AM. Modeling Spin-Forbidden Monomer Self-Initiation Reactions in Spontaneous Free-Radical Polymerization of Acrylates and Methacrylates. The Journal of Physical Chemistry A. 2014;118(40):93108. $\leq \mathrm{DOI}>$. 
39. Verma P, Perera A, Bartlett RJ. Increasing the applicability of DFT I: Non-variational correlation corrections from Hartree-Fock DFT for predicting transition states. Chemical Physics Letters. 2012;524:10-5. <DOI>.

40. Maeda S, Harabuchi Y, Ono Y, Taketsugu T, Morokuma $\mathrm{K}$. Intrinsic reaction coordinate: Calculation, bifurcation, and automated search. International Journal of Quantum Chemistry. 2015;115(5):258-69. <DOI>.
41. Wubbels GG. Use of the Bell-Evans-Polanyi Principle to predict regioselectivity of nucleophilic aromatic photosubstitution reactions. Tetrahedron Letters. 2014;55(36):5066-9. <DOI>.

42. Mayo FR, Lewis FM. Copolymerization. I. A Basis for Comparing the Behavior of Monomers in Copolymerization; The Copolymerization of Styrene and Methyl Methacrylate. Journal of the American Chemical Society. 1944;66(9):1594-601. <DOI>.

43. Odian G. Principles of Polymerization [Internet]. Hoboken, NJ, USA: John Wiley \& Sons, Inc. ; 2004. <DOI >. 


\section{SUPPORTING INFORMATION}

\section{Kinetic Study of the Free Radical Copolymerization of Methyl Methacrylate with Perfluorooctyl Ethyl Methacrylate by Quantum Computational Approach}

Ramazan Katircia and Salih Ozbay ${ }^{b *}$

a Department of Metallurgical and Materials Engineering, Sivas University of Science and Technology, 58000, Sivas, Turkey

${ }^{b}$ Department of Chemical Engineering, Sivas University of Science and Technology, 58000, Sivas, Turkey

Table S1. Thermodynamic parameters computed at $298 \mathrm{~K}$ temperature and 1 atm pressure in the gas phase (PBE0 function and def2-TZVP basis set).

\begin{tabular}{|c|c|c|}
\hline & $\Delta G($ TS-react) (cal) & $k$ (rate) $\mathrm{s}^{-1}$ \\
\hline R--MMA & 134795.9732 & $9.48348773 \mathrm{E}-87$ \\
\hline R-MMA--MMA & 191331.3701 & $3.40388261 \mathrm{E}-128$ \\
\hline R-2MMA---MMA & 253066.1944 & $1.88530918 \mathrm{E}-173$ \\
\hline R-- FOEMA & 423292.0851 & $0.00000000 \mathrm{E}+00$ \\
\hline R- FOEMA -- FOEMA & 803668.3095 & $0.00000000 \mathrm{E}+00$ \\
\hline R-2 FOEMA -- FOEMA & 1159976.621 & $0.00000000 \mathrm{E}+00$ \\
\hline R- FOEMA ---MMA & 483062.7089 & $0.00000000 \mathrm{E}+00$ \\
\hline R-MMA--- FOEMA & 483461.3283 & $0.00000000 \mathrm{E}+00$ \\
\hline R- FOEMA -MMA---MMA & 460561.1927 & $0.00000000 \mathrm{E}+00$ \\
\hline R- FOEMA -MMA--- FOEMA & 767284.4602 & $0.00000000 \mathrm{E}+00$ \\
\hline R-MMA- FOEMA ---MMA & 558585.622 & $0.00000000 \mathrm{E}+00$ \\
\hline R-MMA- FOEMA --- FOEMA & 870388.8911 & $0.00000000 \mathrm{E}+00$ \\
\hline
\end{tabular}

Table S2. Thermodynamic parameters computed at $350 \mathrm{~K}$ temperature and 1 atm pressure in the gas phase (PBE0 function and def2-TZVP basis set).

\begin{tabular}{|c|c|c|}
\hline & $\Delta G$ (TS-react) (cal) & $k$ (rate) $\mathrm{s}^{-1}$ \\
\hline R--MMA & 136429.3997 & $4.62900018 \mathrm{E}-73$ \\
\hline R-MMA--MMA & 189728.7037 & $2.40518257 \mathrm{E}-106$ \\
\hline R-2MMA---MMA & 250298.7436 & $3.60065233 \mathrm{E}-144$ \\
\hline R-- FOEMA & 423853.5596 & $1.49590195 \mathrm{E}-252$ \\
\hline R- FOEMA -- FOEMA & 800609.6047 & $0.00000000 \mathrm{E}+00$ \\
\hline R-2 FOEMA -- FOEMA & 1156812.665 & $0.00000000 \mathrm{E}+00$ \\
\hline R- FOEMA ---MMA & 484992.2149 & $9.88661068 \mathrm{E}-291$ \\
\hline R-MMA--- FOEMA & 481709.1224 & $1.10987083 \mathrm{E}-288$ \\
\hline
\end{tabular}




\begin{tabular}{|c|c|c|}
\hline R- FOEMA -MMA---MMA & 556443.4876 & $0.00000000 \mathrm{E}+00$ \\
\hline R- FOEMA -MMA--- FOEMA & 862918.3969 & $0.00000000 \mathrm{E}+00$ \\
\hline R-MMA- FOEMA ---MMA & 555915.6346 & $0.00000000 \mathrm{E}+00$ \\
\hline R-MMA- FOEMA --- FOEMA & 867352.8704 & $0.00000000 \mathrm{E}+00$ \\
\hline
\end{tabular}

Table S3. The preliminary study results in the different function and basis set.

\begin{tabular}{|c|c|c|c|}
\hline File name & Job & opt & freq \\
\hline aibn_opt-freq & B3LYP 6-31G(d,p) opt freq & -530.683898777583 & + \\
\hline aibn_opt-freq_m062x & $\begin{array}{c}\text { M062X 6-31G(d,p) opt } \\
\text { numfreq }\end{array}$ & -530.789754769161 & + \\
\hline aibn_opt-freq_PBE & PBE $6-31 G(d, p)$ opt freq & -530.342724848908 & + \\
\hline r-aibn_opt-freq_b3lyp & B3LYP 6-31G(d,p) opt freq & -210.605002312145 & + \\
\hline r-aibn_opt-freq_m062x & $\begin{array}{c}\mathrm{m062 \times} 6-31 \mathrm{G}(\mathrm{d}, \mathrm{p}) \text { opt } \\
\text { numfreq }\end{array}$ & -210.635285657286 & + \\
\hline r-aibn_opt-freq_pbe & pbe $6-31 G(d, p)$ opt freq & -210.459817022986 & + \\
\hline MMA_g1_opt-freq_b3lyp & B3LYP 6-31G(d,p) opt freq & -345.573917406707 & + \\
\hline $\begin{array}{l}\text { MMA_g1_opt- } \\
\text { freq_m062x }\end{array}$ & $\begin{array}{c}\text { M062X 6-31G }(d, p) \text { opt } \\
\text { numfreq }\end{array}$ & -345.619493292794 & + \\
\hline MMA_g1_opt-freq_pbe & PBE 6-31G(d,p) opt freq & -345.348240266100 & - \\
\hline MMA_g2_opt-freq_b3lyp & B3LYP $6-31 G(d, p)$ opt freq & -345.588611162832 & $\begin{array}{c}-158.92,- \\
99.61\end{array}$ \\
\hline $\begin{array}{l}\text { MMA_g2_opt- } \\
\text { freq_m062x }\end{array}$ & $\begin{array}{l}\text { M062X 6-31G }(d, p) \text { opt } \\
\text { numfreq }\end{array}$ & -345.638040898266 & + \\
\hline MMA_g2_opt-freq_pbe & PBE $6-31 G(d, p)$ opt freq & -345.366536159985 & - \\
\hline $\begin{array}{l}\text { r-MMA_g1_opt- } \\
\text { freq_b3lyp }\end{array}$ & B3LYP 6-31G(d,p) opt freq & -556.191975070327 & -16.68 \\
\hline $\begin{array}{l}\text { r-MMA_g1_opt- } \\
\text { freq_b3lyp_g1 }\end{array}$ & B3LYP $6-31 G(d, p)$ opt freq & -556.191975062726 & -36.59 \\
\hline $\begin{array}{l}\text { r-MMA_g1_opt- } \\
\text { freq_b3lyp_g2 }\end{array}$ & B3LYP $6-31 G(d, p)$ opt freq & -556.191974040997 & + \\
\hline $\begin{array}{l}\text { r-MMA_g1_opt- } \\
\text { freq_m062x }\end{array}$ & $\begin{array}{l}\text { M062X 6-31G }(d, p) \text { opt } \\
\text { numfreq }\end{array}$ & -556.288521310507 & + \\
\hline r-MMA_g1_opt-freq_pbe & PBE $6-31 G(d, p)$ opt freq & -555.833081894961 & + \\
\hline $\begin{array}{l}\text { r-2MMA_g1_opt- } \\
\text { freq_b3lyp }\end{array}$ & B3LYP $6-31 G(d, p)$ opt freq & -862.529154688673 & + \\
\hline $\begin{array}{l}\text { r-2MMA_g1_opt- } \\
\text { freq_m062x }\end{array}$ & $\begin{array}{l}\text { M062X 6-31G(d,p) opt } \\
\text { numfreq }\end{array}$ & -862.683756120450 & + \\
\hline $\begin{array}{l}\text { r-2MMA_g1_opt- } \\
\text { freq_pbe }\end{array}$ & PBE $6-31 G(d, p)$ opt freq & -861.982881250647 & + \\
\hline $\begin{array}{l}\text { r-3MMA_g1_opt- } \\
\text { freq_b3lyp }\end{array}$ & B3LYP $6-31 G(d, p)$ opt freq & -1168.839909183060 & + \\
\hline $\begin{array}{l}\text { r-3MMA_g1_opt- } \\
\text { freq_m062x }\end{array}$ & $\begin{array}{l}\text { M062X 6-31G(d,p) opt } \\
\text { numfreq }\end{array}$ & -1169.058152291700 & + \\
\hline $\begin{array}{l}\text { r-3MMA_g1_opt- } \\
\text { freq_pbe }\end{array}$ & PBE 6-31G(d,p) opt freq & -1168.109343076010 & -6.27 \\
\hline $\begin{array}{l}\text { r-4MMA_g1_opt- } \\
\text { freq_b3lyp }\end{array}$ & B3LYP 6-31G(d,p) opt freq & -1475.142105875250 & + \\
\hline $\begin{array}{l}\text { r-4MMA_g1_opt- } \\
\text { freq_m062x }\end{array}$ & $\begin{array}{c}\text { M062X 6-31G }(d, p) \text { opt } \\
\text { numfreq }\end{array}$ & -1475.422366291610 & + \\
\hline r-4MMA_g1_opt- & PBE $6-31 G(d, p)$ opt freq & -1474.222309328250 & + \\
\hline
\end{tabular}




\begin{tabular}{|c|c|c|c|}
\hline freq_pbe & & & \\
\hline $\begin{array}{l}\text { r-5MMA_g1_opt- } \\
\text { freq_b3lyp }\end{array}$ & B3LYP 6-31G(d,p) opt freq & -1781.421693099380 & + \\
\hline $\begin{array}{l}\text { r-5MMA_g1_opt- } \\
\text { freq_m062x }\end{array}$ & $\begin{array}{l}\text { M062X 6-31G(d,p) opt } \\
\text { numfreq }\end{array}$ & -1781.780927190650 & -62.38 \\
\hline $\begin{array}{l}\text { r-5MMA_g1_opt- } \\
\text { freq_pbe }\end{array}$ & PBE $6-31 G(d, p)$ opt freq & -1780.325191210910 & 19.71 \\
\hline tm_g1_opt-freq_b3lyp & B3LYP $6-31 G(d, p)$ opt freq & -2385.588424993400 & -159.81 \\
\hline $\begin{array}{c}\text { tm_g1_opt- } \\
\text { freq_b3lyp_g1 }\end{array}$ & B3LYP 6-31G(d,p) opt freq & -2385.590759104760 & 15.76 \\
\hline tm_g1_opt-freq_m062x & $\begin{array}{c}\text { M062X 6-31G(d,p) opt } \\
\text { numfreq }\end{array}$ & -2385.797913196300 & -14.91 \\
\hline tm_g1_opt-freq_pbe & PBE 6-31G(d,p) opt freq & -2384.210973070660 & -151.44 \\
\hline r-tm_g1_opt-freq_b3lyp & B3LYP 6-31G(d,p) opt freq & -2596.216012481470 & + \\
\hline $\begin{array}{l}\text { r-tm_g1_opt- } \\
\text { freq_m062x }\end{array}$ & $\begin{array}{c}\text { M062X 6-31G(d,p) opt } \\
\text { numfreq }\end{array}$ & -2596.470088206310 & + \\
\hline r-tm_g1_opt-freq_pbe & PBE $6-31 G(d, p)$ opt freq & -2594.700783056990 & -10.13 \\
\hline $\begin{array}{l}\text { r-2tm_g1_opt- } \\
\text { freq_b3lyp_duz }\end{array}$ & B3LYP $6-31 G(d, p)$ opt freq & -4981.820703723510 & + \\
\hline r-3tm_g1_freq_b3lyp & B3LYP 6-31G(d,p) numfreq & -7367.426722815420 & + \\
\hline $\begin{array}{c}\text { r-3tm_g1_opt- } \\
\text { freq_m062x_devam }\end{array}$ & $\begin{array}{c}\text { M062X 6-31G }(d, p) \text { opt } \\
\text { numfreq }\end{array}$ & -7368.137540547900 & Not available \\
\hline r-3tm_g1_opt-freq_pbe & PBE $6-31 G(d, p)$ opt freq & -7363.178413765410 & Not available \\
\hline $\begin{array}{l}\text { r-3tm_g1_opt- } \\
\text { freq_pbe_devam }\end{array}$ & PBE $6-31 G(d, p)$ numfreq & -7363.178413561820 & -56.43 \\
\hline
\end{tabular}

Table S4. Gibbs Free Energy of monomers, radicals and transition states (TS) of molecules.

\begin{tabular}{cc}
\hline & $\begin{array}{c}\text { Gibbs Free Energy } \\
\text { (Eh) }\end{array}$ \\
\hline RA (Radical) & -555.93 \\
RB (Radical) & -2595.63 \\
A (Monomer) & -345.43 \\
B (Monomer) & -2385.13 \\
RA -- A (TS) & -901.33 \\
RB -- B (TS) & -4980.73 \\
RA -- B (TS) & -2941.03 \\
RB -- A (TS) & -2941.03 \\
\hline
\end{tabular}

